\title{
Oral surgery: top tips for primary care
}

\author{
By Kamran Ali' and Ewen McColl²
}

A $s$ extractions will make up the majority of oral surgery procedures in primary care, this article focuses on top tips for performing extractions in a safe and predictable manner, with minimal trauma to the hard and soft tissues. The ultimate aim of all these measures is to improve the experience of patients undergoing extractions.

\section{Patient preparation}

Patient preparation is crucial to ensure safety and although tooth extractions can usually be accomplished without complications, it is best to err on the side of caution. Overestimation of tooth extractions often leads to gratitude whilst underestimation may result in embarrassment and medico-legal consequences:

- Obtain a thorough patient assessment and evaluate any risks of tooth extractions due to comorbidities such as cardiovascular or hepatorenal disease, diabetes, immunosuppression, and concomitant medications such as blood thinners or anti-resorptive drugs. ${ }^{1,2}$ Do not hesitate to discuss your treatment plan with the patient's GP if there are any concerns regarding a patient's medical status

- Carry out a thorough clinical assessment to evaluate access to the tooth, its position in the arch, condition of the crown and note any adjacent restorations which may be at risk of damage during instrumentation for tooth extraction. Consider an appropriate radiograph to obtain a good view of the peri radicular region and identify anatomical challenges (dilaceration, hypercementosis, accessory roots, ankylosis, concrescence and proximity to antral floor or inferior alveolar/mental nerve etc $)^{3}$

- Obtain a valid patient consent through shared decision making. Whilst patient reassurance is paramount, it is equally important to moderate expectations, clearly explain potential risks and resist the temptation to promise too much!

- Always consider the use of a surgical safety checklist to minimise human errors resulting in wrong tooth extractions. ${ }^{4}$

\section{Select appropriate instruments}

A variety of instruments may be used for undertaking tooth extractions including forceps, luxators and elevators. It seems sensible to arrange all instruments which may be possibly required prior to the procedure. Fetching additional instruments during the procedure is time consuming and makes nurses feel pressurised. Moreover, it may compromise cross infection control due to the need to get instruments from drawers and frequent changing of gloves etc. In addition, the dentist may be perceived by the patient to be struggling due to repeated need for additional instruments. Indeed, oral surgeons lay out all the instruments on the surgical tray prior to commencing the procedure.

\section{Prioritise access}

Good access and vision of the surgical site is fundamental to performing any operative procedure including tooth extractions and requires appropriate positioning of the operator and the patient; adequate retraction of soft tissues (lips, cheeks, tongue etc); and excellent lighting conditions throughout the procedure. While the need for good access is obvious, we have often observed clinicians using a position of mechanical disadvantage to approach the patient and ignoring poor soft tissue retraction and lighting, especially when dealing with difficult extractions. Unfortunately, this attitude only makes the procedure more challenging and adds to the stress and frustration of the dentist (and the patient). It is best to focus on maintaining a clear access and vision to the operative site throughout by appropriate positioning, good soft tissue retraction, ensuring unimpeded passage of light and effective suction.

\section{Use your instruments correctly}

Notwithstanding the wide variations in the difficulty levels of tooth extractions encountered in clinical practice and different approaches used by clinicians, it is often helpful to commence tooth extractions with luxators. Unless ankylosed, teeth are anchored in the alveolar bone by the periodontal ligament. Luxators have a sharp blade and can be used as periotomes to sever the periodontal fibres with minimal force. When used appropriately, luxators can often make

\section{'Good access and vision of the surgical site is fundamental to performing any operative procedure including tooth extractions'}

tooth extractions a straightforward job - almost. Next, elevators such as Coupland's can be used to elevate the tooth by engaging it with the elevator blade near the cemento-enamel junction on the buccal aspect. Rotation of the elevator along its long axis may allow elevation of the tooth along its path of withdrawal. Finally, the correct forceps can be used to displace and deliver the tooth securely.

Some of the generic principles applicable during extraction with any type of instruments include:

- Protect the airway securely with a tongue depressor and use high volume suction; this is especially important using elevators as tooth fragments may be dislodged into the airway with potentially adverse consequences

- Use the non-dominant hand effectively to retract the soft tissues; support the alveolus (and mandible) to minimise the risk of bone fracture, and to obtain tactile feedback during tooth movements

- Never lean on the patient - no knees on the patient's chest please!

- Use a buccal approach and avoid working across the arch 
41. The extraction instrument must rest only on the tooth to be extracted without any impingement on the soft tissues and avoid any force on adjacent teeth or restorations

- Avoid excessive apical force on maxillary posterior teeth in close proximity to the antral floor and mandibular molars in close proximity to the inferior alveolar nerve

- Always use a controlled force and see what the tooth wants to do. If the tooth is stubborn and no movement is achieved, revaluate the radiographs to rule out anatomical variations (dilaceration, hypercementosis, ankylosis, accessory roots etc). Consider a surgical approach if within the competency of the operating dentist; otherwise, it may be best to stop the procedure and consider a specialist referral.

\section{Provide good post-extraction management}

Several measures are required to achieve uneventful healing following a tooth extraction: ${ }^{5}$

- After a successful tooth extraction, avoid the temptation to place a bite pack within the next microsecond. Palpate the socket walls to rule out the presence of any sharp bone and smooth with a bone file or rose-head surgical bur if required. Look carefully down the socket and use saline irrigation and suction to clear away any debris in the socket and reduce the microbial load. These measures are important to ensure uncomplicated healing of the extraction socket

- Achieve haemostasis prior to discharging the patient and never send the patient home with a bite pack in situ. A pressure pack placed directly over the extraction socket and held securely with the bite for ten minutes is usually adequate to achieve haemostasis.
Patients must avoid talking to the dentist/nurse during this time as it may compromise haemostasis. Patients on blood thinners may require additional local measures to achieve haemostasis ${ }^{1}$

- Provide written and verbal post extraction instructions re pain control, postoperative bleeding, oral hygiene, diet and things to avoid (smoking, alcohol, heavy exercise). Patients must be instructed to avoid active spitting/rinsing for at least 24 hours after extractions as it may dislodge the blood clot in the socket, compromise healing and possibly risking the development of alveolar osteitis

- Provide an emergency contact number and arrange a follow-up for complicated extractions.

Finally, dentists may consider additional training to develop their extraction skills specially to master safe and effective use of luxators and elevators and to carry out surgical tooth extractions. It may be worth exploring opportunities to work some sessions at local oral surgery units in primary and secondary care or local dental schools. Given the opportunity, such exposure may provide invaluable experience to dentists interested in improving their oral surgery skills.

\section{References}

1. Toole J, McKenna G, Smyth J. Managing patients at risk of medication related complications requiring dental extractions in primary care. Prim Dent J 2020; 9: 54-58

2. Wilmott $S$, Yates J, Pretty I A. Dental extractions in primary care for patients at risk of MRONJ. Br Dent J 2021; doi: 10.1038/s41415-021-3674-8.

3. Hupp M R, Ellis J R, Tucker E. Contemporary oral and maxillofacial surgery. 6th edition. pp 92-112. Missouri: Elsevier, 2008.

4. Dargue A, Fyfe E, French K et al. The impact of wrong-site surgery on dental undergraduate teaching: a survey of UK dental schools. Eur J Dent Educ 2021; 25: 670-678.

5. Sambrook P J, Goss A N. Contemporary exodontia. Aust Dent J 2018; DOI: 10.1111/adj.12586

\section{Government dragging heels on overseas dentists left unable to practise}

The British Dental Association (BDA) has called on Ministers and the General Dental Council (GDC) to deliver rapid change to laws governing tests for overseas dentists, after COVID restrictions have left thousands of applicants waiting to pass registration exams to practise in the UK.

The BDA has warned that a three-month consultation on necessary legal changes - launched on 7 February - will mean little chance of parliamentary time until the autumn at the earliest, leaving an incredibly narrow window until mutual recognition of qualifications for EU dentists ends in December.

Due to the pandemic, the Overseas Registration Exam (ORE) has not taken place since January 2020. In response to a parliamentary question, Minister Maria Caulfield MP revealed that there are over 2,000 applicants currently waiting for an opportunity to sit these exams. Under existing arrangements, most of these dentists will end up waiting years before being allowed a chance to practise in the UK and due to outdated regulations, many won't be allowed to work in the NHS.

The GDC recently told 132 candidates who had already passed the first part of their ORE that they cannot currently be allocated a place to sit Part 2 as - due to no exams taking place for two years - they have exceeded the permitted waiting time of five years from the first attempt at Part 1.

In messages to these candidates on 3 December 2021, the GDC said there was nothing it could do given the legislation it works to. The consultation that has now been published addresses the imminent issues for these candidates but the timescales involved mean further delays to meaningful change. The consultation had been expected since the middle of 2021 and it is not clear why it has taken so long to get off the ground. The BDA will consider all proposals and provide relevant feedback but stressed government must act quickly on feedback.

The BDA has been overwhelmed by messages from candidates citing desperation, suicidal thoughts and immense anger at the current situation. It has heard from dentists who have postponed having children because of the delays and who have had to work in other roles such as minicab drivers, security guards, busboys and supermarket shelf stackers to make ends meet.

The BDA had pressed both the GDC and Health Secretary, Sajid Javid to work at pace to resolve the situation and bring down the backlog awaiting examinations and improve the processes which allow overseas dentists to work in the NHS.

Patients in England face profound access problems, with over 38 million NHS appointments lost since the first lockdown and with 